\title{
Article \\ Nanofluid Transport through a Complex Wavy Geometry with Magnetic and Permeability Effects
}

\author{
Muhammad Saleem Iqbal ${ }^{1}$, Abuzar Ghaffari ${ }^{2}$ (D), Arshad Riaz ${ }^{2, *(D)}$, Irfan Mustafa ${ }^{3}$ and Muhammad Raza $^{4}$ \\ 1 Department of Mathematics, Islamabad College for Boys G-6/3, Islamabad 44000, Pakistan; \\ msiqbal366@gmail.com \\ 2 Department of Mathematics, Division of Science and Technology, University of Education, Lahore 54770, \\ Pakistan; abuzar.ghaffari@ue.edu.pk \\ 3 Department of Mathematics, Allama Iqbal Open University, H-8, Islamabad 44000, Pakistan; \\ irfan.mustafa@aiou.edu.pk \\ 4 Department of Mathematics, Comsats University, Islamabad 44000, Pakistan; \\ muhammadraza01214@gmail.com \\ * Correspondence: arshad-riaz@ue.edu.pk
}

Citation: Iqbal, M.S.; Ghaffari, A.; Riaz, A.; Mustafa, I.; Raza, M. Nanofluid Transport through a Complex Wavy Geometry with Magnetic and Permeability Effects. Inventions 2022, 7, 7. https:// doi.org/10.3390/inventions7010007

Academic Editor:

Goshtasp Cheraghian

Received: 19 October 2021

Accepted: 15 December 2021

Published: 25 December 2021

Publisher's Note: MDPI stays neutral with regard to jurisdictional claims in published maps and institutional affiliations.

Copyright: (C) 2021 by the authors. Licensee MDPI, Basel, Switzerland. This article is an open access article distributed under the terms and conditions of the Creative Commons Attribution (CC BY) license (https:// creativecommons.org/licenses/by/ $4.0 /)$.

\begin{abstract}
The current article incorporates the numerical investigation of heat exchange rate and skin friction carried out through nanofluid saturated with thermally balanced porous medium over a rough horizontal surface that follows the sinusoidal waves. The effects of the external magnetic field are discussed by managing the magnetic field strength applied normally to the flow pattern. The occurring partial differential governing equations are grasped through a strong numerical scheme of the Keller box method (KBM) against the various parameters. The findings are elaborated through tables and diagrams of velocity, temperature, skin friction, Nusselt number, streamlines, and heat lines. The percentage increase in Nusselt number and coefficient of skin friction over the flat and wavy surface is calculated which leads to the conclusion that the copper $(\mathrm{Cu})$ nanoparticles are better selected as compared to the silver $(\mathrm{Ag})$ for heat transfer enhancement. It is also evident from sketches that the current analysis can be used to enhance the surface drag force by means of nanoparticles. It is a matter of interest that the magnetic field can be used to manage the heat transfer rate in such a complicated surface flow. The current readings have been found accurate and valid when compared with the existing literature.
\end{abstract}

Keywords: copper nanofluid; silver nanofluid; Darcy frame; wavy surface; Keller box method; Nusselt number

\section{Introduction}

Heat flow augmentation is a major interest in industry, scientific, and engineering research. The role of heat transfer has much importance in the natural system and all devices such as chemical processing, heat exchangers, high-performance gas turbines, energy devices, and general manufacturing. The involvement of nanofluid makes enhancement in Nusselt number which adds an essential contribution in the discipline of mechanical sciences such as solar energy systems, thermal storage systems, nuclear reactors' cooling, reducing the temperature of electronic devices and turbomachinery, etc. The discussion on effective thermal transfer by the addition of nanoparticles in the based fluid has been given in the previous studies [1-5].

The analysis of heat exchange flows across the porous space has due importance for its vast implementation in the field of technological processes such as packed bed reactors, recovery of petroleum resources, heat insulation, drying technology, and nuclear waste repository, etc. In high temperatures and friction, nanofluid can be used in cooling the machinery and equipment.

Nakayama and Hossain [6] have studied the aspects of the porous medium and used an integral approach to handle its theoretical results. Singh et al. [7] analyzed that 
porosity of the channel can be employed to handle the thermal transfer enhancement. They encountered these results while studying the convective flow problems under the magnetic influence for various fieldstrengths. Ram et al. [8] disclosed that in the porous structure, the heat flow rate can be controlled by thermal source/sink resource. The study of porosity factors under the influence of magnetic environment is also invested by Cheng [9]. He used a surface of wavy texture while his theoretical investigation and integral approach is used for this purpose. Yih [10] performed a theoretical study of convective flow in the porous medium along the truncated having the surface of the wavy texture. He considered both cases of VMF/VHF (variable heat/mass flux) and VWC/VWT (variable wall temperature/concentration).

Hassan and Rashidi [11] reported micropolar flow in a porous channel and found that by minimizing the averaged residual error, we can obtain the possible optimal value of the convergence-control parameters which may give the fastest convergent series. Rashidi et al. [12] discussed second-grade fluid flow through a porous medium and obtained the solution by the DTM-Padé method and revealed that the DTM-Padé is an excellent method for solving the problems that have boundary conditions at infinity. Bhatti et al. [13] studied MHD Williamson nanofluid flow over a porous shrinking sheet. It is observed that entropy profile increases for all the physical parameters. Freidoonimehr et al. [14] investigated 3D nanofluid flow in a rotating channel on a lower permeable stretching porous wall and showed that the downward motion of the upper plate augments the forward flow, whereas the upward motion reverses the flow.

In references [15-19] different well-known researchers performed theoretical studies on the topic of the porous medium by incorporating various trends and different geometries. Ghaffarpasand [20] presented double-diffusive natural convection flow for two-dimensional skewed enclosure with an external magnetic field and found that Lorentz force due to applied magnetic field generates assisting/opposing flow for Lewis number/buoyancy ratio parameter.

Ram et al. [21] studied unsteady magnetic nanofluid flow past a rotating plate and concluded that the Nusselt number is enhanced by increasing the Prandtl number. Akram [22] explored 2D (two-dimensional) channel flow for fourth-grade nanofluid and evaluated the analytic behavior of the study. Golshokooh et al. [23] investigated the impact of hybrid silica Nano-sized particles on enhancing oil reproduction in a 3D void having regime and showed that more oil produced in water emulsion from hybrid silica nanoparticles in comparison to polymer alone. Sheikholeslami and Rokni [24] disclosed the impact of radiation on heat transport in a porous enclosure and found that temperature gradient rises by incrementing Darcy number and radiation parameter. Zeeshan et al. [25] inspected the natural convection MHD flow of nanofluid past a porous enclosure. They used HAM for the investigation of flow and transfer rates. Hakeem et al. [26] inspected MHD flow across a stretching/shrinking surface using second order partial slip in the non-Darcy porous regime and concluded lower branch solution for shrinking does not exist at the high magnetic field.

Recently, Sheikholeslami et al. [27] scrutinized the impact of thermally radiated $\mathrm{Fe}_{3} \mathrm{O}_{4}$ ethylene glycol nanofluid EHD transport in a porous space and noticed that the thermal transfer rate flourishes by strengthening radiation parameter. Ghaffarpasanda and Fazelia [28] analyzed induced MHD (magnetohydrodynamics) mixed convection in porous parallelograms enclosure and a decrease in Nusselt number is observed with the reduction of porous permeability.Rana et al. [29] studied MHD (magnetohydrodynamics) fluid flow due to unsteady moving surface in nanofluid using the KKL (Koo-Kleinstreuer-Li) model and observed that the temperature profile of hydrodynamic flow is less than that of MHD (magnetohydrodynamics) flow. Bhuvaneswari et al. [30] probed the impact of viscous energy loss on second-grade fluid behavior and noticed that the Nusselt number improved/reduced with the intensification of suction/injection factors and Biotnumber whereas, the heat exchange rate is reduced with the strengthening of heat generation/absorption factor and Eckert number. Alsagri et al. [31] reported MHD nanofluid 
flow in a duct with viscous forces effects saw seen that the skin friction constant reduces by strengthening magnetic force. Sheikholeslami and Rokni [32] inspected the characteristics of Brownian induced motion on MHD natural convection nanofluid flow in a porous container and saw that Nusselt number reduces for the larger impact of Hartmann number.Some more related studies on wavy geometry can be seen in Refs. [33-42].

The above literature review bears witness that very little concentration has been imported to the area of nanofluids through a wavy enclosure. The analysis of nanofluid over a moving wave generating surface along with the porous space has not been analyzed yet. The present study is the extension work of Reddy et al. [43]. Reddy et al. [43] examined the problem for regular fluid (base fluid) while in the current analysis we analyzed for nanofluid by considering the copper and silver nanoparticle. The results are calculated numerically by means of Keller box scheme. Thepresent investigation reveals heat transfer enhancement by incorporating different nanoparticles. This examines different volume fractions ranging from 0 to $5 \%$. Results of interests such as velocity profile, temperature profile, skin friction factor, and Nusselt number against including parameters are revealed to elaborate the effect of nanoparticles.

\section{Materials and Methods}

We considered the hydromagnetic steam of nanofluid over a rough moving surface having sinusoidal nature $\left(y=\bar{S}(\bar{x})=\bar{\alpha} \operatorname{Sin}\left(\frac{\pi \bar{x}}{l}\right)\right)$ placed horizontally in the $x$-direction. The medium is considered as porous, where $\bar{x}$ represents the streamwise location along $x$-axis, $l$ is the wavelength and $\bar{\alpha}$ represents the amplitude. The constant magnitude of the magnetic field is supposed in the direction of $\bar{y}$ under the constraint of low magnetic Reynold's number. The porous medium is assumed to contain copper and silver particles. In the presence of these particles, the heat transfer effects are examined. In addition, the wall temperature is considered higher than the ambient one. The velocity component $\bar{u}$ and $\bar{v}$ are taken along $\bar{x}$ and $\bar{y}$ direction as shown in Figure $1 \mathrm{a}, \mathrm{b}$ and the possible boundary layer pattern is described in right-side frame.

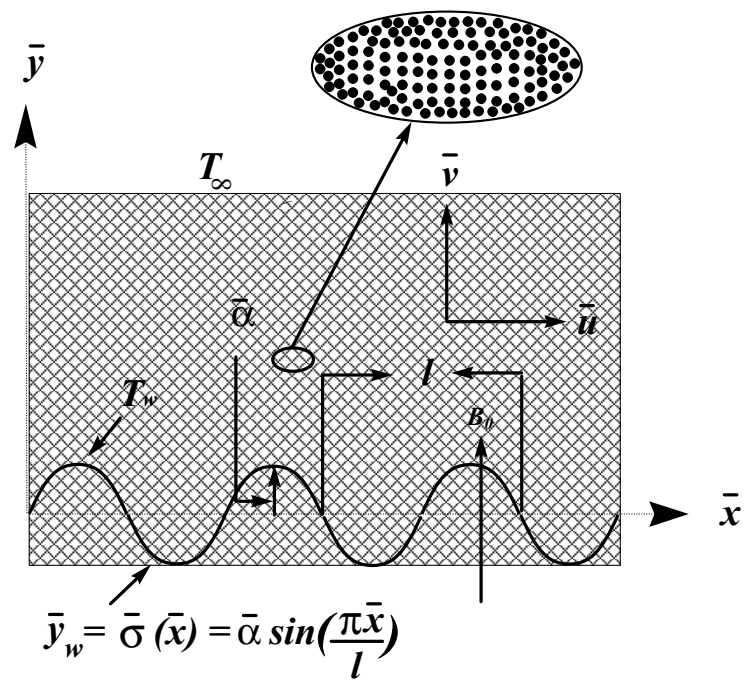

(a)

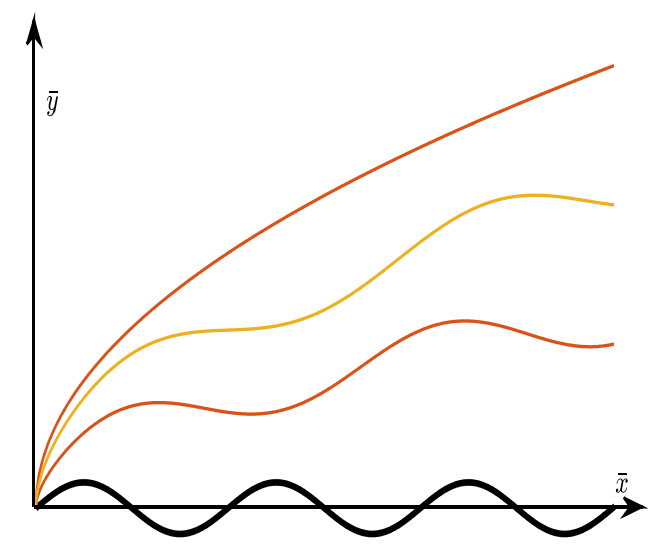

(b)

Figure 1. $(\mathbf{a}, \mathbf{b})$ The geometry of the considered problem.

To effectively identify the influence of nanoparticles in heat flow phenomena, a suitable model is required. There are several theoretical and experimental models based on single and multi-phase nanofluid models. In single-phase nanofluid models Tiwari and Das [32] is a good model presented in the literature to examine the phenomenon of nanofluid convective transport. It is one of the popular nanofluid models which represent the 
enhanced nanofluid material properties. According to this model, the constitutive equations in vector form can be written as [43-46]:

$$
\begin{gathered}
\nabla . \mathrm{V}=0 \\
(\mathrm{~V} . \nabla) \mathrm{V}=-\left(1 / \rho_{\mathrm{nf}}\right) \nabla \mathrm{p}+v_{\mathrm{nf}} \nabla^{2} \mathrm{~V}-\left(v_{\mathrm{nf}} / \mathrm{K}\right) \mathrm{V}-\left(\sigma_{\mathrm{nf}} \mathrm{B}_{0} / \rho_{\mathrm{nf}}\right) \mathrm{V} \\
\mathrm{V} . \nabla \mathrm{T}=\alpha_{\mathrm{nf}} \nabla^{2} \mathrm{~T}
\end{gathered}
$$

where $V=[\bar{u}(\bar{x}, \bar{y}), \bar{v}(\bar{x}, \bar{y})]$ is the velocity field, $\mathrm{T}$ is for temperature, $\sigma_{\mathrm{nf}}$ is for nanofluids' electric conductivity, $\overline{\mathrm{p}}$ is for pressure, $\varrho_{\mathrm{nf}}$ for nanofluids' density, $\mathrm{B}_{0}$ is used to represent magnetic field strength, $\mathrm{T}_{\infty}$ isambient temperature and $\nabla^{2}$ is Laplacian operator. The boundary conditions are:

$$
\begin{gathered}
\bar{y}=\bar{S}(\bar{x}): \bar{u}=U, \bar{u}=0, T=T_{\omega}, \text { for all } \bar{x}>0 \\
\bar{y} \rightarrow \infty: \bar{u}=0, \bar{p}=p_{\infty}, T=T_{\infty} \text { for all } \bar{x}>0 .
\end{gathered}
$$

Incorporating the following transformations [43]

$$
\begin{gathered}
\xi=x=\frac{\bar{x}}{l}, y=\frac{\bar{y}}{l}, S=\frac{\bar{S}(\bar{x})}{l}, u=\frac{\bar{u}}{U^{\prime}}, y=\frac{(\bar{y}-\bar{S}(\bar{x})) \sqrt{R e}}{l}, v=\frac{\sqrt{R e}}{U}\left(\bar{v}-S_{\xi} \bar{u}\right), \\
p=\frac{\bar{p}-p_{\infty}}{\rho_{f} U^{2}}, R e=\frac{U l}{v_{f}}, D a^{-1}=\frac{v_{f} l}{K U}, M=\frac{\sigma_{f} B_{0}^{2} l}{\rho_{f} U}, \eta \sqrt{\xi}=y, \Omega=\sqrt{1+S_{\tilde{\xi}}^{2}}, \\
u=\frac{\partial \psi}{\partial x}, v=-\frac{\partial \psi}{\partial y}, \psi(\xi, \eta)=\sqrt{\xi} f(\xi, \eta), \theta(\xi, \eta)=\frac{T-T_{\infty}}{T_{w}-T_{\infty}},
\end{gathered}
$$

where $K$ is the permeability of saturated porous medium. Darcy number is the representation of cross-sectional area of porous medium Versace radiative effects of the permeability of the porous medium.

We obtain the following set of PDEs

$$
\begin{gathered}
\frac{\Omega^{2}}{A_{1}} f^{\prime \prime \prime}+\frac{1}{2} f f^{\prime \prime}-\xi\left(\frac{D a^{-1}}{A_{2}}+\frac{M A_{4}}{A_{2} \Omega^{2}}\right) f^{\prime}-\xi \frac{\Omega_{\xi}}{\Omega}\left(f^{\prime}\right)^{2}=\xi\left[f^{\prime} \frac{\partial f^{\prime}}{\partial \xi}-f^{\prime \prime} \frac{\partial f}{\partial \xi}\right], \\
\frac{A \Omega^{2}}{A_{3} \operatorname{Pr}} \theta^{\prime \prime}+\frac{1}{2} f \theta^{\prime}=\xi\left[f^{\prime} \frac{\partial \theta}{\partial \xi}-\theta^{\prime} \frac{\partial f}{\partial \xi}\right],
\end{gathered}
$$

where prime symbol "'" denotes differentiation w. r. t $\eta$. The combined parameters $A, A_{1}, A_{2}, A_{3}$ and $A_{4}$ are

$$
\begin{gathered}
A=\frac{\kappa_{\mathrm{nf}}}{\kappa_{\mathrm{f}}}, A_{1}=(1-\phi)^{2.5}\left((1-\phi)+\phi\left(\rho_{p} / \rho_{f}\right)\right), A_{2}=\left((1-\phi)+\phi\left(\rho_{p} / \rho_{f}\right)\right), \\
A_{3}=\left((1-\phi)+\phi\left(\left(\rho c_{p}\right)_{p} /\left(\rho c_{f}\right)_{f}\right)\right), A_{4}=1+\frac{3\left(\left(\sigma_{\mathrm{p}} / \sigma_{\mathrm{f}}\right)-1\right) \phi}{\left(\left(\sigma_{\mathrm{p}} / \sigma_{\mathrm{f}}\right)+2\right)-\phi\left(\left(\sigma_{\mathrm{p}} / \sigma_{\mathrm{f}}\right)-1\right)}
\end{gathered}
$$

Boundary conditions are

$$
\begin{gathered}
\mathrm{f}(\xi, 0)=0, \mathrm{f}^{\prime}(\xi, 0)=1, \theta(\xi, 0)=1, \\
\mathrm{f}^{\prime}(\xi, \infty)=0, \theta(\xi, \infty)=0 .
\end{gathered}
$$

The local Nusselt number and skin friction coefficient are defined as

$$
\mathrm{C}_{\mathrm{f}}=\mathrm{C}_{\mathrm{fx}} \operatorname{Re}^{1 / 2}=\frac{\mathrm{x}^{-3 / 2}}{\Omega^{3 / 2}(1-\phi)^{2.5}} \mathrm{f}^{\prime \prime}(\xi, 0), \mathrm{Nu}=\mathrm{Nu}_{\mathrm{x}} \operatorname{Re}^{-1 / 2}=-\frac{\mathrm{x}^{1 / 2}}{\Omega^{1 / 2}} \frac{\kappa_{\mathrm{nf}}}{\kappa_{\mathrm{f}}} \theta^{\prime}(\xi, 0) .
$$

The nanofluid physical properties can be found inRefs. [31-39]. 


\subsection{Numerical Results}

The governing non-similar set of partial differential Equations (6), (7) and (9) is tackled by Keller box strategy (for details see $[47,48]$ ) incorporation with the implicit finite difference scheme. By using Keller box implicit finite difference technique the partial differential Equations (6) and (7) are initially reduced to a system of 1st order differential equations by choosing new variables:

$$
f^{\prime}=g, g^{\prime}=q, \theta^{\prime}=p, \varphi^{\prime}=d,
$$

Block tri-diagonal procedure is applied after writing in matrix vector form which is then solved by using block tri diagonal procedure, which contains two sweeps, i.e., forward and backward. Before comparison, we preformed runs of various step sizes for $\eta$ and $\xi$ parameters and observed that an accuracy achieved for numerical values of $-C f \& N u$ are up to $10^{-4}$. For the validation purpose the comparison of archived solution is made with the studies present in the literature as given in Table 1 . This table illustrates that the numerical results of physical quantities i.e., $C_{f x} R e_{x}^{1 / 2}$ and $N u_{x} R e_{x}^{-1 / 2}$ agree very close with the previously published data.

Table 1. Validation of results for different $M$ when $P r=0.7, \alpha=\phi=D a^{-1}=0$.

\begin{tabular}{|c|c|c|c|c|c|c|c|c|c|}
\hline \multirow[t]{3}{*}{$\xi$} & \multicolumn{3}{|c|}{$-C_{f}=-f^{\prime \prime}(\xi, 0)$} & \multicolumn{2}{|c|}{$-C_{f}=-f^{\prime \prime}(\xi, 0)$} & \multicolumn{2}{|c|}{$-C_{f}=-f^{\prime \prime}(\xi, 0)$} & \multicolumn{2}{|c|}{$N u=-\theta^{\prime}(\xi, 0)$} \\
\hline & $M=0.0$ & $\mathrm{M}=0.01$ & & $M=0.1$ & & $M=0.5$ & & $M=0.5$ & $\mathrm{M}=\mathbf{0 . 0}$ \\
\hline & {$[44,45,49]$} & Present & [50] & Present & [50] & Present & [50] & Present & {$[44,45,49]$} \\
\hline 0.0 & $\begin{array}{c}(0.44375) \\
(0.4438) \\
(0.4439)\end{array}$ & 0.443749 & 0.443751 & 0.443749 & 0.443751 & 0.443749 & 0.443751 & 0.349242 & $\begin{array}{c}(0.349242) \\
(0.3492) \\
(0.3509)\end{array}$ \\
\hline 0.1 & - & 0.444417 & 0.444421 & 0.450417 & 0.450467 & 0.476872 & 0.476966 & 0.341787 & - \\
\hline 0.2 & - & 0.445084 & 445091 & 0.457057 & 0.457160 & 0.509420 & 0509627 & 0.334250 & - \\
\hline 0.3 & - & 0.445750 & 0.445760 & 0.463676 & 0.463831 & 0.541427 & 0.541753 & 0.326620 & - \\
\hline 0.4 & - & 0.446417 & 0.446429 & 0.470272 & 0.470480 & 0.572896 & 0.573368 & 0.318894 & - \\
\hline 0.5 & - & 0.447083 & 0.447098 & 0.476847 & 0.477107 & 0.603834 & 0.604488 & 0.311072 & - \\
\hline 0.6 & - & 0.447749 & 0.447767 & 0.483400 & 0.483712 & 0.634244 & 0.635127 & 0.303153 & - \\
\hline 0.7 & - & 0.448415 & 0.448435 & 0.489931 & 0.490296 & 0.664133 & 0.665291 & 0.295137 & - \\
\hline 0.8 & - & 0.449081 & 0.449104 & 0.496441 & 0.496858 & 0.693506 & 0.694984 & 0.287028 & - \\
\hline 0.9 & - & 0.449746 & 0.449772 & 0.502928 & 0.503398 & 0.722369 & 0.724201 & 0.278827 & - \\
\hline 1.0 & - & 0.450411 & 0.450440 & 0.509394 & 0.509917 & 0.750730 & 0.752938 & 0.270545 & - \\
\hline
\end{tabular}

\subsection{Grid Independence Test}

Before comparison, we performed runs of various step sizes for $\eta$ and $\xi$ parameters and observed that an accuracy achieved for numerical values of $-C f \& N u$ are up to $10^{-4}$ as listed in as given in Table 2. An equal step size 0.005, 3000 and 400 grid points were taken in $\eta$ and $\xi$-direction. We assume infinity at 20 on which we found sufficiently stable and accurate solution.

For the validation purpose, the comparison of the archived solution is made with the studies present in the literature as given in Table 1. This table illustrates that the numerical results of physical quantities i.e., $C_{f x} R e_{x}^{1 / 2}$ and $N u_{x} R e_{\mathrm{x}}^{-1 / 2}$ agree very closely with the previously published data. 
Table 2. For regular fluid $(\varphi=0.0)$ gird independence test at $\operatorname{Pr}=7.0, D a=\alpha=M=0.1$.

\begin{tabular}{cccc}
\hline $\begin{array}{c}\text { Grid Points in } \boldsymbol{\eta} \\
\text { Direction with Fixed } \boldsymbol{\eta}=20\end{array}$ & $\begin{array}{c}\text { Grid Points in } \xi \\
\text { Direction with Fixed } \xi=0.5\end{array}$ & $-\boldsymbol{C f}$ & $\boldsymbol{N u}$ \\
\hline 50 & 5 & 3.1871 & 0.5677 \\
\hline 100 & 10 & 3.1802 & 0.5546 \\
\hline 200 & 20 & 3.1777 & 0.5517 \\
\hline 400 & 40 & 3.1766 & 0.5509 \\
\hline 800 & 80 & 3.1761 & 0.5506 \\
\hline 1600 & 160 & 3.1758 & 0.5509 \\
\hline 3200 & 320 & 3.1757 & 0.5505 \\
\hline 4000 & 400 & 3.1757 & 0.5505 \\
\hline
\end{tabular}

\section{Results and Discussion}

Influence of different emerging dimensionless parameters on physical quantities $C_{f}$ and $\mathrm{Nu}$ are captured through graphs. In these graphs, solid and dashed lines represent copper and silver nanoparticles. The effects of magnetic number $M$, the amplitude representative $\alpha$, fraction parameter of nanoparticle $\phi$ and inverse Darcy number $D a^{-1}$ on $C_{f}$ and $\mathrm{Nu}$ have been displayed in Figures 2-5 for two nanoparticles copper $(\mathrm{Cu})$ and silver $(A g)$. Figures 2 and 3 demonstrate the effect of copper and silver nanoparticles on the skin friction parameter $\left(C_{f}\right)$ and the Nusselt number $(N u)$, when $M=0,1$, 3. It is found that at the surface of the wavy wall, the skin friction increases as the magnetic field strength becomes stronger. From this phenomenon we can say that near the wavy surface wall, magnetic field resists the fluid motion and magnetic strength oppose the flow which results in an enhancement in drag force. We have also noticed that at the surface of the wavy wall, the heat flow rate reduces due to which heated wavy wall takes a long time to transfer the heat in the liquid at ambient temperature. One can also imagine that for the case of silver more increment in the value of skin friction is achieved as compared to copper, but the behavior is quite opposite for the heat transfer rate. These outcomes provide that silver nanoparticles under the magnetic effects enhance the fluid movement resistance closer to the surface of the heated wavy wall and we obtain enhancement in the skin friction measure. Whereas the heat flow intensity in case of silver nanoparticles under the magnetic effects slow down the process of the heat travelling from the thermal wavy boundary to the ambient fluid temperature.

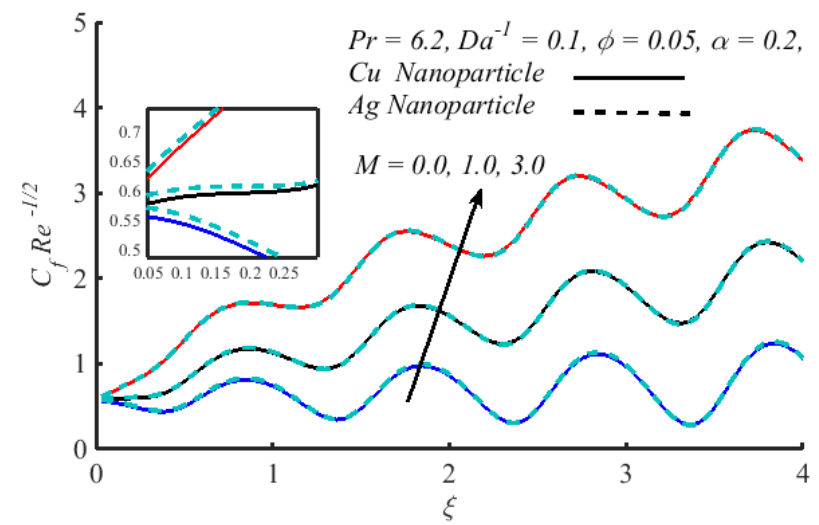

Figure 2. Magnetic effect on skin friction. 


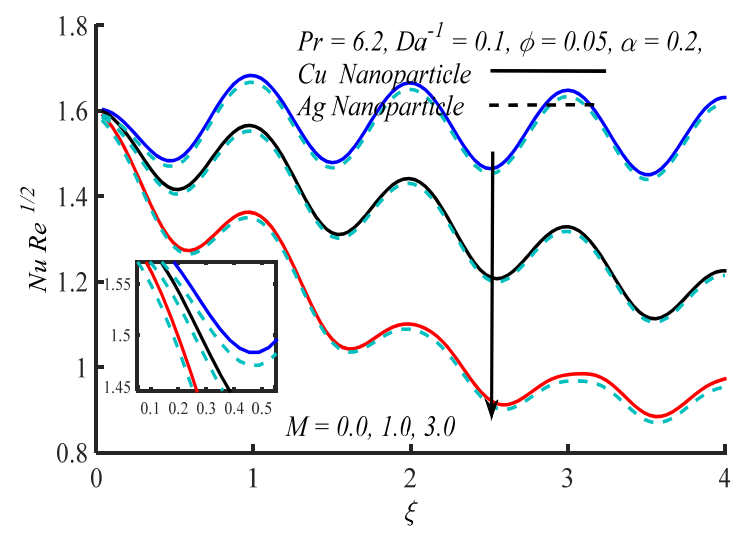

Figure 3. Magnetic impact on Nusselt number.

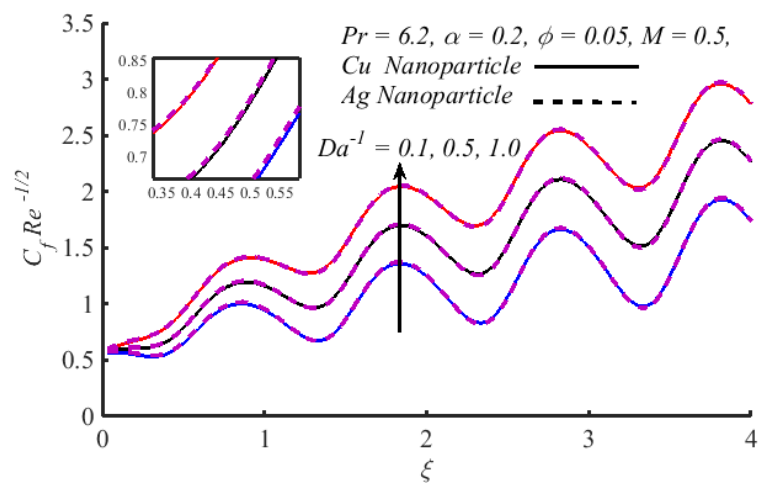

Figure 4. Porosity effect on skin friction.

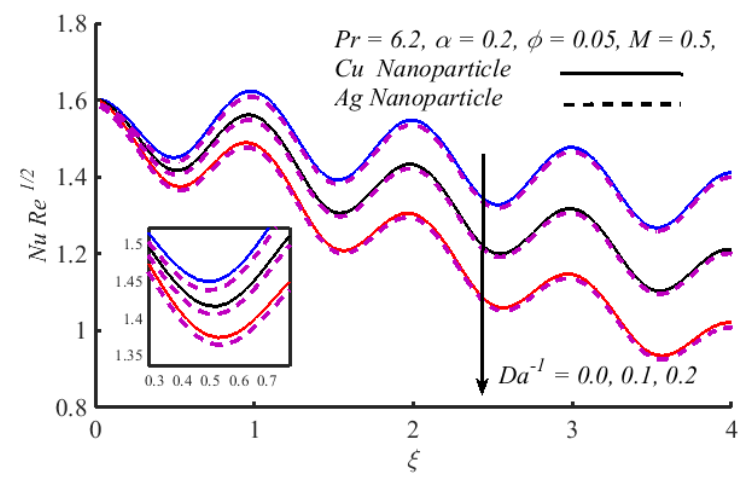

Figure 5. Porosity effect on the Nusselt number.

The effect of inverse Darcy number on $C_{f}$ and $N u$ is shown in Figures 4 and 5. Skin friction is observed as an increasing function and Nusselt number is observed a decreasing function of inverse Darcy number. This is because the increase in inverse Darcy number results in a reduction in permeability of the porous medium. It is also noted that copper $\mathrm{Cu}$ ) nanoparticles remains dominant in case of inverse Darcy number as compared to silver (Ag) nanoparticles. On the other hand, the situation gets reversed when we look at the graphs for $\mathrm{Nu}$. It is also noted that silver and copper particles gives a minor difference especially in the case of inverse Darcy number in Figure 4. In Figure 5, it is noted that there is a notable variation between copper particles and silver particles concentration. Moreover, when noted from the said graphs, there is no variation on the start of the domain but it becomes significant from left to right surface which reflects the decrease in porosity measures as we travel away from the wavy surface. On the whole domain, the curves are showing wavy characteristics which later on start diverging. 
Figure 6 illustrates the influence of Pr on thermal thickness, it is seen that thermal thickness reduces with the intensification of $\operatorname{Pr}$, which induces that the incrementing $\operatorname{Pr}$ corresponds to the strengthening of the heat diffusivity and as a result, thermal thickness reduces significantly. It is also seen here that for $\operatorname{Pr}=0.62$, the curve is having negative slope but as we provide larger values to the Prandtl number, the curves are having larger slopes tending to infinity and vertical curves are obtained. Moreover, the curves are coming closer to each other which may collide or change their characteristics with much larger impact of Prandtl number. It may be due to the fact that Prandtl number being the ratio of specific heat and thermal conductivity depends upon the nature of the material of nanoparticles which results that the nanoparticles with larger thermal conductivity increases the rate of heat transfer in the flow. Figure 7 show the impact of $\mathrm{Da}^{-1}$ on thermal distribution. The figures depict that augmentation of $D a^{-1}$ the fluid temperature rises within the boundary layer. This is due to the fact that the permeability of the porous medium reduces with the increase in inverse Darcy number.

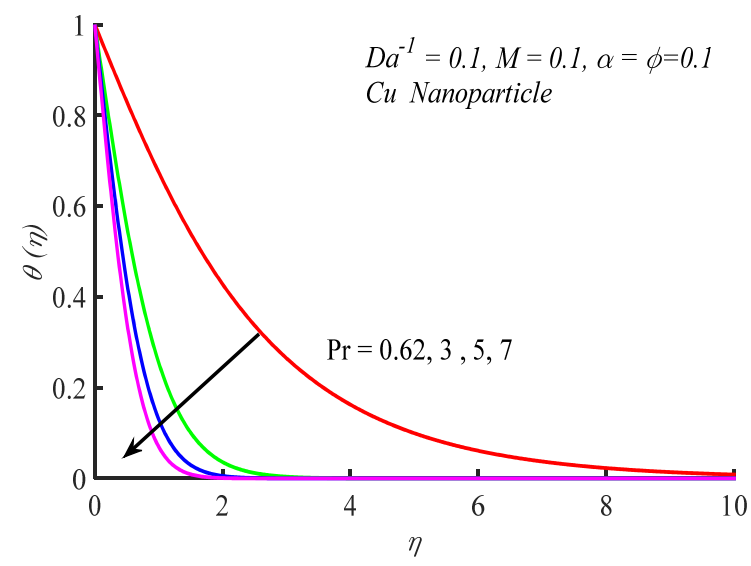

Figure 6. Alteration of thermal thickness against Pr.

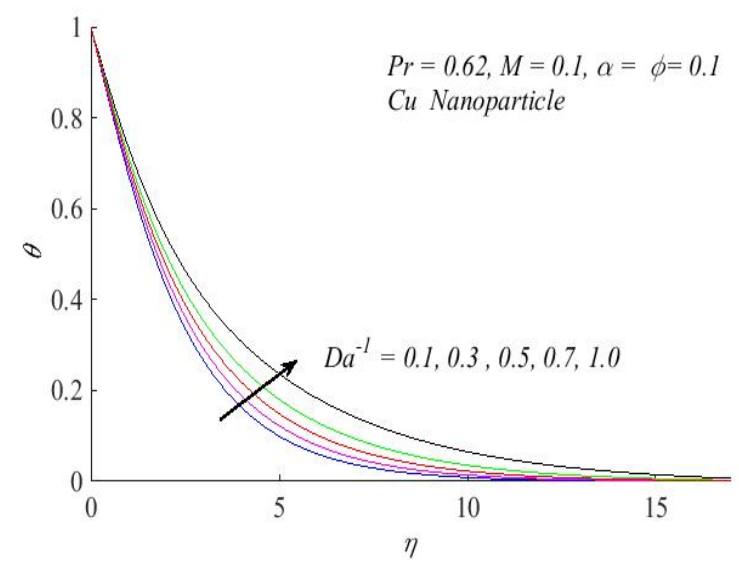

Figure 7. Variation of thermal thickness against $D a^{-1}$.

The streamlines are presented in Figures 8 and 9. The graphs are drawn specifically for inverse Darcy and magnetic parameters in case of copper nanoparticles. It is seen that the magnetic field can be used to strengthen the copper nanoparticle's characteristics to enhance the heat transfer effects. It is also further seen that that increasing the values of inverse Darcy parameter, boost up the heat transfer rate. On the other hand, we can depict from here that the intensity of porosity in the medium is inversely affecting the rate of thermal exchange. Therefore, we can enhance the rate of thermal transfer by reducing the impact of porosity in the surface. On the other hand, magnetic field is in favor of 
heat transfer rate so it can be used in various physical mechanisms where large thermal exchange rate is needed.

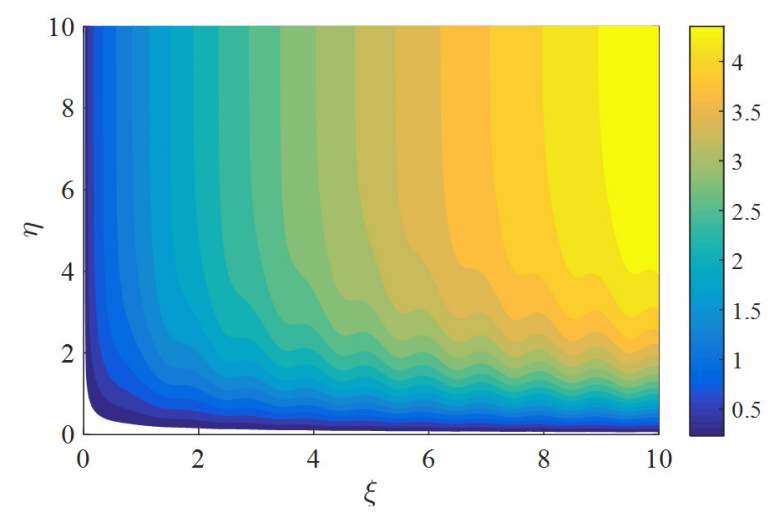

Figure 8. Streamlines for $\mathrm{M}=0, \alpha=0.2, D a^{-1}=0.01, \operatorname{Pr}=6.2, \phi=0.1$ with copper nanoparticles.

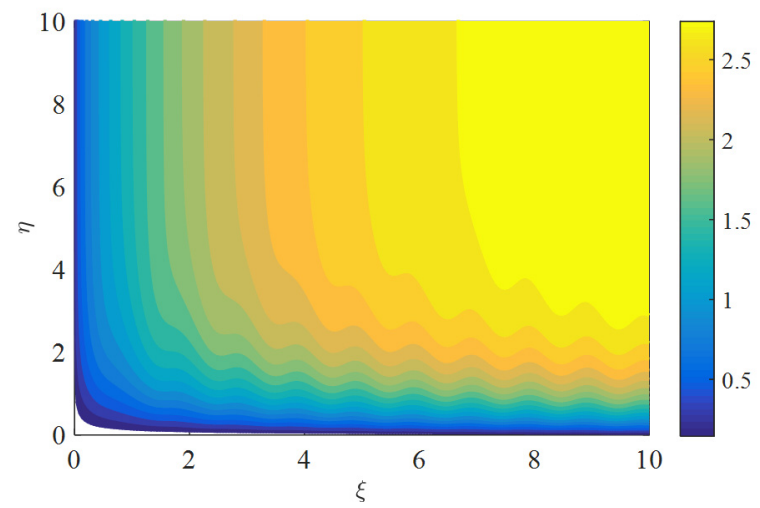

Figure 9. Streamlines for $\mathrm{M}=0.2, \alpha=0.2, D a^{-1}=0.01, \operatorname{Pr}=6.2, \phi=0.1$ with copper nanoparticles.

\section{Concluding Remarks}

This analysis is carried out theoretically to mainly study the heat exchange rate due to nanoparticles saturations in the base fluid (water) over the continuously moving surface of the wavy texture through a porous medium. The magnetic force of constant strength under the assumption of low magnetic Reynold's number is imposed in an orthogonal direction. The porous medium is assumed to contain copper and silver particles. In the presence of these particles, the heat transfer effects are examined. It is also measured that the wall temperature is higher than the ambient temperature. This physical problem is transformed into mathematical equations, which are treated by a well-known numerical algorithm of Kellerbox. The pattern of discussion is arranged through Tables and diagrams. This analysis evaluates that:

1. Near the wavy surface wall, the magnetic field resists the fluid traveling and magnetic strength oppose the flow which results in an enhancement in drag force.

2. Silver nanoparticles under the magnetic effects enhance the fluid motion resistance closer to the heated wavy wall and we obtain enhancement in the skin friction coefficient.

3. Heat transfer rate in case of silver nanoparticles under the magnetic effects slow down the process of the heat transfer from the heated wavy wall to the ambient fluid temperature.

4. Flow rate near the boundary layer portion can be controlled by the nanoparticles' concentration.

5. The process of the heat exchange from the thermal wavy wall can manage by the nanoparticles' concentration. 
6. Wavy amplitude increment results in the enhancement of skin friction $\left(C_{f}\right)$ at the crest of the wavy surface but at the trough the skin friction decreases.

7. Wavy amplitude increment results in the heat transfer rate $(\mathrm{Nu})$ enhancement at the crest of the wavy surface but at the trough, the heat transfer rate $(\mathrm{Nu})$ decreases.

Author Contributions: Conceptualization, M.S.I. and A.G.; methodology, A.R.; software, I.M.; validation, A.R. writing —original draft preparation, A.G.; writing—review and editing, I.M.; investigation and supervision, M.R. All authors have read and agreed to the published version of the manuscript.

Funding: This research received no external funding.

Acknowledgments: The authors are grateful to the Higher Education Commission of Pakistan for the support.

Conflicts of Interest: The authors declare no conflict of interest.

\section{Nomenclature}

\begin{tabular}{|c|c|}
\hline Symbols & Names with Units \\
\hline$A, A_{1}, A_{2}, A_{3}, A_{4}$ & Constant material parameters \\
\hline$B_{0}$ & Magnetic induction, Tesla $\mathrm{NA}^{-1} \mathrm{~m}^{-2}$ \\
\hline$c_{p}$ & Specific Heat, \\
\hline$g$ & Acceleration of gravity, $\mathrm{m}^{2} \mathrm{~s}^{-1}$ \\
\hline $\mathrm{Da}^{-1}$ & Inverse of Darcy number \\
\hline$k$ & Thermal Conductivity, $\mathrm{Wm}^{-1} \mathrm{~K}^{-1}$ \\
\hline$l$ & Characteristic length of the wavy plate, $\mathrm{m}$ \\
\hline$M$ & Magnetic number \\
\hline $\bar{p}$ & Dimensionless Pressure, $\mathrm{Nm}^{-2}$ \\
\hline$p$ & Dimensionless pressure \\
\hline $\operatorname{Pr}$ & Prandtl Number \\
\hline $\bar{S}$ & Wavy surface \\
\hline$T$ & Local temperature, $K$ \\
\hline$(\bar{u}, \bar{v})$ & Dimensional Velocity component in $(x, y)$ direction \\
\hline$(u, v)$ & Dimensionless velocity component in the $X$ direction \\
\hline$(\bar{x}, \bar{y})$ & Dimensional coordinates \\
\hline$(x, y)$ & Dimensionless coordinates \\
\hline$\alpha^{*}$ & Thermal diffusivity, $\mathrm{m}^{2} \mathrm{~s}$ \\
\hline$\alpha$ & Amplitude of the wavy surface \\
\hline$\beta$ & Coefficient of thermal expansion, $\mathrm{K}^{-1}$ \\
\hline$\Omega$ & Wavy parameter \\
\hline$(\xi, \eta)$ & new computational independent variables \\
\hline$v$ & Kinematic viscosity, $\mathrm{m}^{2} \mathrm{~s}^{-1}$ \\
\hline$\sigma$ & Electrical conductivity, $\Omega^{-1} \mathrm{~m}^{-1}$ \\
\hline$\psi$ & Dimensionless Stream function Subscript \\
\hline$\rho$ & Local density, $\mathrm{kgm}^{-3}$ \\
\hline$\theta$ & Dimensionless temperature \\
\hline$\phi$ & Solid Volume Friction \\
\hline$\mu$ & Dynamic viscosity, $\mathrm{kgm}^{-1} \mathrm{~s}^{-1}$ \\
\hline \multicolumn{2}{|l|}{ subscripts } \\
\hline$f$ & Base fluid \\
\hline$n f$ & Nanofluid \\
\hline$p$ & Nanoparticle \\
\hline$w$ & Condition at the surface \\
\hline$\infty$ & Condition far away from surface \\
\hline
\end{tabular}




\section{References}

1. Choi, S.U.S. Enhancing Conductivity Thermal of Fluids with Nanoparticles; ASME Publications: New York, NY, USA, $1995 ;$ pp. 99-105.

2. Hosseini, S.M.; Safaei, M.R.; Estellé, P.; Jafarnia, S.H. Heat transfer of water-based carbon nanotube nanofluids in the shell and tube cooling heat exchangers of the gasoline product of the residue fluid catalytic cracking unit. J. Therm. Anal. Calorim. 2019, 140, 351-362. [CrossRef]

3. Bhatti, M. Biologically Inspired Intra-Uterine Nanofluid Flow under the Suspension of Magnetized Gold (Au) Nanoparticles: Applications in Nanomedicine. Inventions 2021, 6, 28. [CrossRef]

4. Nayak, M.; Shaw, S.; Khan, M.I.; Pandey, V.; Nazeer, M. Flow and thermal analysis on Darcy-Forchheimer flow of copper-water nanofluid due to a rotating disk: A static and dynamic approach. J. Mater. Res. Technol. 2020, 9, 7387-7408. [CrossRef]

5. Ellahi, R.; Hussain, F.; Abbas, S.A.; Sarafraz, M.M.; Goodarzi, M.; Shadloo, M.S. Study of Two-Phase Newtonian Nanofluid Flow Hybrid with Hafnium Particles under the Effects of Slip. Inventions 2020, 5, 6. [CrossRef]

6. Nakayama, A.; Hossain, M. An integral treatment for combined heat and mass transfer by natural convection in a porous medium. Int. J. Heat Mass Transf. 1995, 38, 761-765. [CrossRef]

7. Singh, H.; Ram, P.; Kumar, A. A study of the effect of chemical reaction and radiation absorption on MHD convective heat and mass transfer flow past a semi-infinite vertical moving plate with time dependent suction. Int. J. Appl. Math Mech. 2011, 7, 38-58.

8. Ram, P.; Kumar, A.; Singh, H. The effect of chemical reaction and heat transfer on MHD flow of viscous fluid past a moving isothermal vertical porous plate with time dependent suction. Int. J. Theor. Appl. Mech. 2011, 6, 241-254.

9. Cheng, C.Y. Effect of a magnetic field on heat and mass transfer by natural convection from vertical wavy surfaces in porous media-an integral approach. Int. Comm. Heat Mass Transf. 1999, 26, 935-943. [CrossRef]

10. Yih, K.A. Coupled heat and mass transfer by free convection over a truncated cone in porous media: VWT/VWC or VHF/VMF. Acta Mech. 1999, 137, 83-97. [CrossRef]

11. Hassan, H.; Rashidi, M.M. An analytic solution of micropolar flow in a porous channel with mass injection using homotopy analysis method. Int. J. Numer. Method Heat Fluid Flow 2014, 24, 419-437. [CrossRef]

12. Rashidi, M.; Hayat, T.; Keimanesh, T.; Yousefian, H. A study on heat transfer in a second-grade fluid through a porous medium with the modified differential transform method. Heat Transf.-Asian Res. 2012, 42, 31-45. [CrossRef]

13. Bhatti, M.M.; Abbas, T.; Rashidi, M.M. Numerical study of entropy generation with nonlinear thermal radiation on magnetohydrody-namics non-Newtonian nanofluid through a porous shrinking sheet. J. Magn. 2016, 21, 468-475. [CrossRef]

14. Freidoonimehr, N.; Rostami, B.; Rashidi, M.M.; Momoniat, E. Analytical Modelling of Three-Dimensional Squeezing Nanofluid Flow in a Rotating Channel on a Lower Stretching Porous Wall. Math. Probl. Eng. 2014, 2014, 692728. [CrossRef]

15. Cheng, C.-Y. Natural convection heat and mass transfer near a vertical wavy surface with constant wall temperature and concentration in a porous medium. Int. Commun. Heat Mass Transf. 2000, 27, 1143-1154. [CrossRef]

16. Maleki, H.; Safaei, M.R.; Togun, H.; Dahari, M. Heat transfer and fluid flow of pseudo-plastic nanofluid over a moving permeable plate with viscous dissipation and heat absorption/generation. J. Therm. Anal. Calorim. 2018, 135, 1643-1654. [CrossRef]

17. Rees, D.A.S.; Pop, I. A Note on Free Convection Along a Vertical Wavy Surface in a Porous Medium. J. Heat Transf. 1994, 116, 505-508. [CrossRef]

18. Cheng, C.Y. Double diffusion from a vertical wavy surface in a porous medium saturated with a non-Newtonian fluid with constant wall temperature and concentration. Int. Comm. Heat Mass Transf. 2007, 34, 285-294. [CrossRef]

19. Kumar, B.V.R. Free convection in a non-Darcian wavy porous enclosure. Int. J. Eng. Sci. 2003, 41, 1827-1848. [CrossRef]

20. Ghaffarpasand, O. Unsteady double-diffusive natural convection with Soret and Dufour effects inside a two-sided lid-driven skewed enclosure in the presence of applied magnetic field. Sci. Iran. Trans. B Mech. Eng. 2017, 25, 3526-3530. [CrossRef]

21. Ram, P.; Joshi, V.K.; Makinde, O.D.; Kumar, A. Convective boundary layer flow of magnetic nanofluids under the influence of geo-thermal viscosity. Defect Diffus. Forum 2018, 387, 296-307. [CrossRef]

22. Akram, S. Nanofluid effects on peristaltic transport of a fourth grade fluid in the occurrence of inclined magnetic field. Sci. Iran. Trans. F Nanotechnol. 2016, 23, 1502-1516. [CrossRef]

23. Golshokooh, S.; Ramazani, S.A.; Hekmatzadeh, M. Investigating the effect of hybrid silica nanoparticles-copolymer on increasing oil recovery in a three dimensional porous media. Sci. Iran. 2017, 24, 3466-3475.

24. Sheikholeslami, M.; Rokni, H.B. Numerical simulation for impact of Coulomb force on nanofluid heat transfer in a porous enclosure in presence of thermal radiation. Int. J. Heat Mass Transf. 2018, 118, 823-831. [CrossRef]

25. Zeeshan, A.; Ellahi, R.; Hassan, M. Magneto hydrodynamic flow of water/ethylene glycol based nanofluid with natural con-vection through a porous medium. Eur. Phys. J. Plus 2014, 129, 261. [CrossRef]

26. Hakeem, A.A.; Ganesh, N.V.; Ganga, B. Heat transfer of non-Darcy MHD flow of nanofluid over a stretching/shrinking surface in a thermally stratified medium with second order slip model. Sci. Iran. Trans. F Nanotechnol. 2015, 22, $2766-2784$.

27. Ganji, D.D.; Sheikholeslami, M.; Li, Z.; Hosseinnejad, R. Numerical simulation of thermal radiative heat transfer effects on Fe3O4-Ethylene glycol nanofluid EHD flow in a porous enclosure. Sci. Iran. Trans. B Mech. Eng. 2019, 26, 2766-2784. [CrossRef]

28. Ghaffarpasand, O.; Fazeli, D. Numerical analysis of MHD mixed convection flow in a parallelogramic porous enclosure filled with nanofluid and in the presence of magnetic field induction. Sci. Iran. Trans. F Nanotechnol. 2018, 25, 1789-1807.

29. Rana, S.; Nawaz, M.; Qureshi, I.H. Numerical study of hydrothermal characteristics in nanofluid using KKL model with Brownian motion. Sci. Iran. Trans. F Nanotechnol. 2019, 26, 1931-1943. 
30. Eswaramoorthi, S.; Sivasankaran, S.; Rajan, S.; Alshomrani, A.S.; Bhuvaneswari, M. Effects of viscous dissipation and convective heating on convection flow of a second grade liquid over a stretching surface: Analytical and numerical study. Sci. Iran. Trans. $B$ Mech. Eng. 2019, 26, 1350-1357. [CrossRef]

31. Alsagri, A.S.; Hassanpour, A.; Alrobaian, A.A. Simulation of MHD nanofluid flow in existence of viscous dissipation by means of ADM. Case Stud. Therm. Eng. 2019, 14, 100494. [CrossRef]

32. Sheikholeslami, M.; Rokni, H.B. Magnetic nanofluid flow and convective heat transfer in a porous cavity considering Brownian motion effects. Phys. Fluids 2018, 30, 012003. [CrossRef]

33. Sheikholeslami, M.; Ganji, D.D.; Javed, M.Y.; Ellahi, R. Effect of thermal radiation on magnetohydrodynamics nanofluid flow and heat transfer by means of two phase model. J. Magn. Magn. Mater. 2014, 374, 36-43. [CrossRef]

34. Zeeshan, A.; Shehzad, N.; Ellahi, R.; Alamri, S.Z. Convective Poiseuille flow of Al2O3-EG nanofluid in a porous wavy channel with thermal radiation. Neural Comput. Appl. 2017, 30, 3371-3382. [CrossRef]

35. Zeeshan, A.; Majeed, A.; Akram, M.J.; Alzahrani, F. Numerical investigation of MHD radiative heat and mass transfer of nanofluid flow towards a vertical wavy surface with viscous dissipation and Joule heating effects using Keller-box meth-od. Math. Comput. Simul. 2021, 190, 1080-1109. [CrossRef]

36. Sheremet, M.A.; Pop, I. Natural convection in a wavy porous cavity with sinusoidal temperature distributions on both side walls filled with a nanofluid: Buongiorno's mathematical model. J. Heat Transf. 2015, 137, 072601. [CrossRef]

37. Sheremet, M.; Pop, I.; Bachok, N. Effect of thermal dispersion on transient natural convection in a wavy-walled porous cavity filled with a nanofluid: Tiwari and Das' nanofluid model. Int. J. Heat Mass Transf. 2016, 92, 1053-1060. [CrossRef]

38. Ahmed, S.E.; Mahdy, A. Laminar MHD natural convection of nanofluid containing gyrotactic microorganisms over vertical wavy surface saturated non-Darcian porous media. Appl. Math. Mech. Engl. Ed. 2016, 37, 471-484. [CrossRef]

39. Mahdy, A.; Ahmed, S.E. Laminar Free Convection Over a Vertical Wavy Surface Embedded in a Porous Medium Saturated with a Nanofluid. Transp. Porous Media 2011, 91, 423-435. [CrossRef]

40. Cheng, C.Y. Non similar solutions for double diffusive convection near a frustum of a wavy cone in porous media. App. Math. Comp. 2007, 194, 156-167. [CrossRef]

41. Cheng, C.-Y. Double-diffusive natural convection along a vertical wavy truncated cone in non-Newtonian fluid saturated porous media with thermal and mass stratification. Int. Commun. Heat Mass Transf. 2008, 35, 985-990. [CrossRef]

42. Cheng, C.-Y. Combined heat and mass transfer in natural convection flow from a vertical wavy surface in a power-law fluid saturated porous medium with thermal and mass stratification. Int. Commun. Heat Mass Transf. 2009, 36, 351-356. [CrossRef]

43. Reddy, P.B.A.; Reddy, A.S.; Reddy, N.B. Magnetohydrodynamic boundary layer flow and heat transfer over a continuous moving wavy surface embedded in a porous medium. Int. J. Appl. Math. Mech. 2012, 8, 7-85.

44. Mehmood, A.; Iqbal, M.S.; Mustafa, I. Cooling of Moving Wavy Surface through MHD Nanofluid. Z. Nat. 2016, 71, 583-593. [CrossRef]

45. Hossain, M.A.; Pop, I. Magneto-hydrodynamic boundary layer flow and heat transfer on a continuous moving wavy surface. Act. Mech. 1996, 48, 813-823.

46. Sheikholeslami, M.; Oztop, H.F. MHD free convection of nanofluid in a cavity with sinusoidal walls by using CVFEM. Chin. J. Phys. 2017, 55, 2291-2304. [CrossRef]

47. Cebeci, T.; Bradshaw, P. Physical and Computational Aspects of Convective Heat Transfer; Springer: New York, NY, USA, 1988.

48. Na, T.Y. Computational Methods in Engineering Boundary Value Problems; Academic Press: New York, NY, USA, 1979.

49. Rees, D.A.S.; Pop, I. Boundary layer flow and heat transfer on a continuous moving wavy surface. Acta Mech. 1995, 112, 149-158. [CrossRef]

50. Chiam, T.C. Magnetohydodynamic heat transfer over a non-isothermal stretching sheet. Acta Mech. 1997, 122, 169-179. [CrossRef] 